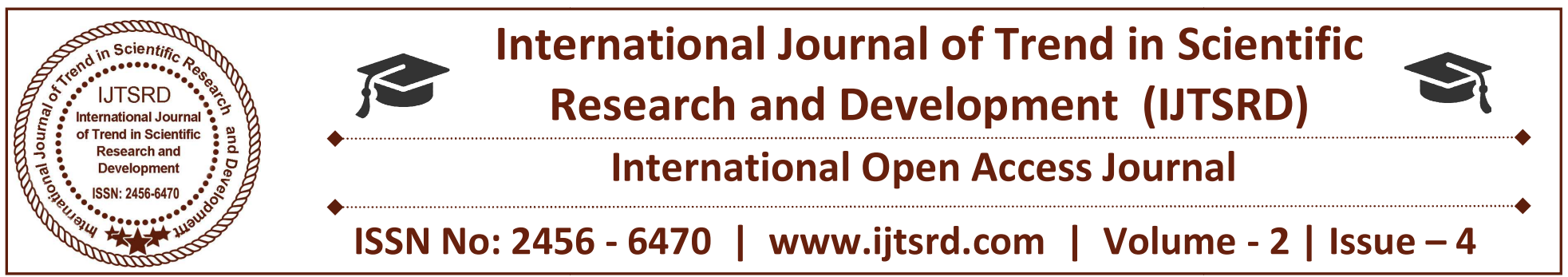

\title{
Single Image Super-Resolution Using Analytical Solution for L2-L2 Algorithm
}

\author{
Varsha Patil ${ }^{1}$, Meharunnisa SP $^{2}$ \\ ${ }^{1}$ Student, ${ }^{2}$ Professor \\ Department of Electronics \& Instrumentation Engineering, \\ Dayananda Sagar College of Engineering, Bengaluru, Karnataka, India
}

\begin{abstract}
This paper addresses a unified work for achieving single image super-resolution, which consists of improving a high resolution from blurred, decimated and noisy version. Single image super-resolution is also known as image enhancement or image scaling up. In this paper mainly four steps are used for enhancement of single image resolution: input image, low sampling the image, an analytical solution and L2 regularization. This proposes to deal with the decimation and blurring operators by their particular properties in the frequency domain, which leads to a fast super-resolution approach. And an analytical solution obtained and implemented for the L2regularization i.e. L2-L2 optimized algorithm. This aims to reduce the computational cost of the existing methods by the proposed method. Simulation results taken on different images and different priors with an advance machine learning technique and conducted results compared with the existing method.
\end{abstract}

Keywords: Single image super-resolution, deconvolution, decimation, analytical solution, L2regularization

\section{INTRODUCTION}

Single image super-resolution (SR) is nothing but the image scaling up or image enhancement. In this the high resolution image can be obtained from the low resolution image. The increased number of pixels within a given image size is called as super resolution (SR). This super-resolution (SR) image consist various information, these information very important for various applications. This super-resolution still an ongoing research with applications in a variety of fields, such as remote sensing [1], video surveillance [2], hyper spectral [3], microwave [4] and medical imaging [5].

The methods in single image SR are classified into three types. The first type is the interpolation based algorithms, bicubic interpolation or adaptive interpolation. These algorithms are simple and easy to implement. And these algorithms are generally very smooth the high frequency details. The second type is learning-based or example-based algorithm. From a given database these algorithms learns about the relations between low resolution and high resolution image patches. These learning-based algorithms almost depend on the training image database. The third type is the reconstruction based algorithm. This algorithm formulates the image super-resolution as reconstruction problem by introducing regularizations into the ill-posed inverse problem.

In this paper, a new approach handles the decimation and blurring operator all together by exploring their basic properties in the frequency domain and aims to reduce the computational cost of these methods by proposing a new approach. It is interesting to note that similar property was explored for multi-frame super resolution. In this report the observed low resolution image assumed to a noisy, sub-sample and blur version of the high resolution image with a spatially invariant blur and derives a closed-form expression for the solution associated with the L2-penalized least-squares SR problem. These referrers as L2-L2 in what underlies the restoration of an image 
contaminated by additive Gaussian noise and used intensively for the single image SR problem. This solution shows to be easily embeddable into an Augmented Lagrangian (AL) framework to handle non-Gaussian priors, which considerably lightens the computational burdens of several existing SR algorithms.

\section{LITERATURE REVIEW}

Jian Sun Jian Sun Zongben Xu Heung-Yeung Shum [10] has proposed Image Super-Resolution using Gradient Profile Prior. In this they suggested a parametric prior describing the shape and the sharpness of the image gradients by an image superresolution move towards using a novel generic image prior - gradient profile prior. Large number of natural images well-read from the gradient profile prior, it provides a restriction on image gradients when it estimates a high resolution image from low resolution image. These gradient restrictions help us to sharpen the information and repress ringing artefacts along with edges.

G. Martin and J. M. Bioucas-Dias [1] has proposed Hyper spectral compressive acquisition in the spatial domain via blind factorization. In this they proposed a new method to perform compressive sensing of hyper spectral images that relies in two characteristics of these data: 1) HSIs admits a low dimensional linear representation; and 2) the coefficients (in the spatial domain) of the linear representations are piecewise smooth, thus compressible [1]. Linear representation is well-read with low computational cost from the compressed measurements was blind by proposed method. The demonstrated in both synthetic and real scenarios was effectiveness of the proposed compressive sensing method. The amount data obtain in this system and transmitters to the ground stations reduces and the bulk of the computation to infer the original data is carried out in the ground stations by using compressive sensing in these systems. In this proposed method gives way very good results yet in low SNR and low measurement rate situations using actual and synthetic data.

I. Yanovsky, B. H. Lambrigtsen, A. B. Tanner, and L. A. Vese [4] has proposed Efficient deconvolution and super-resolution methods in microwave imagery. In this they developed a well-organized deconvolution and super-resolution methodology and be relevant these techniques to reduce image blurring and distortion intrinsic in an opening synthesis method.
These types of a system produce ringing at pointed edges and transitions in the observed meadow. The conservative move towards to suppressing side lobes was to applied linear apodization, which unwanted of degrading spatial resolution side effect. They developed a well-organized total difference minimization technique based on Split Bregman deconvolution which reduces image ringing at the same time as sharpening the image and protects information content. The model is generalized to include up sampling of deconvolved image to a higher resolution network. Their super-resolution methodologies were based on current research in sparse optimization and compressed sensing that leads to unprecedented efficiencies for solving image reconstruction problems.

\section{METHODOLOGY}

In this section, the methodology of this over all proposed method is explained and discussed. The above figure 3.1 block diagram shows the proposed project of each step in that it involves pre-processing steps, image enhancement, image restoration, analytical solution, reconstruction method.

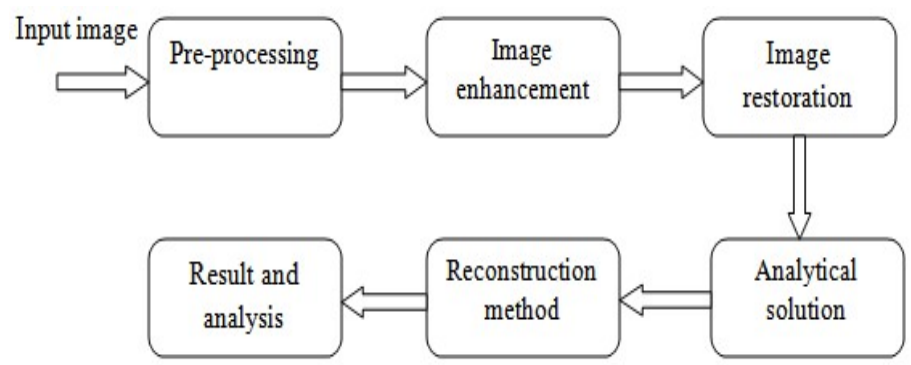

Figure 1 block diagram of proposed method

The RGB (red, gray and blue) images are extracted into the $\mathrm{XYZ}$ co-ordinates and the $\mathrm{Cb}, \mathrm{Cr}$ are the colour channels. These changed RGB images are then upsampling by the Bicubic-interpolation algorithm. In this proposed project 2D Gaussian filter is used with filter size of 7 and the standard deviation of 3 . The Gaussian noises are filtered by the Gaussian low pass filter. Gaussian filter is used eliminate the sharp edges for blurring of the image. The images contaminated by the additive white Gaussian noise. The decimation matrix $\mathrm{S}$ down-testing administrator, while its conjugate transpose $\mathrm{SH}$ interjects the destroyed picture with zeros.

The most computationally affluent portion for the calculation in Theorem 1 is the employment of FFT or IFFT. In aggregate, four FFT and IFFT working outs are prerequisite in our enactment. Associating with 
the innovative problem, the imperative of calculation complexity has diminished suggestively from $\mathrm{O}\left(N_{h}{ }^{3}\right)$ to $\mathrm{O}\left(\mathrm{N}_{\mathrm{h}} \log \mathrm{N}_{\mathrm{h}}\right)$, which consents the investigative solution to be calculated professionally. Note spoken image SR hitches by means of the possessions of $\mathrm{S}$ in the frequency province, where $N_{l}$ minor mediums of size $d \times d$ remained reversed. The complete computational complication of the approaches considered is $\mathrm{O}\left(N_{h} \log N_{h}+N_{h} d^{2}\right)$. Another imperative transformation with our work is that the decomposed SR problem into an up-sampling and a de-blurring stage. The operatives $\mathrm{H}$ and $\mathrm{S}$ were thus measured unconnectedly, thus necessitating two $\mathrm{L}_{2}$ regularizations for the distorted image and the pulverized truth image. On the contrary, this exertion considers the obscuring and down sampling jointly and accomplish the SR in one phase, necessitating individual one regularization stretch for the mysterious image. It is earnest to indication that the anticipated SR explanation can be prolonged to integrate the distorting operator, which can similarly be demonstrated as a BCCB matrix. In the consequence of this segment, two individual occurrences of the $L_{2}$-norm regularization are deliberated, well-defined in the image and gradient provinces, correspondingly.

\section{A. Resolution of the $L_{2}-L_{2}$ delinquent in the image dominion:}

Initially, we deliberate the explicit instance where we convert the input image to frequency domain by adding a noise after applying FFT function given by

$$
\mathrm{Y}=\mathrm{X}^{\mathrm{H}} \text { fft }(\mathrm{X})
$$

This suggests that the objective image is $a$ prior nearby to the image $x$. The image $x$ can be an assessment of the HR image, e.g., an interpolated variety of the experimental image, a reestablished image attained with learning based algorithms. In such circumstance, by means of Theorem 1, the explanation of the problematic is

$$
\text { xest }=\left(B^{\mathrm{H}} * \mathrm{~S}^{\mathrm{H}} * \mathrm{~S} * \mathrm{H}+\text { tau } * \mathrm{I}\right)^{-\mathrm{R}}
$$

Where, $\mathrm{B}$ is shift invariant, $\mathrm{S}$ is the input low sampled image segment, $\mathrm{H}$ is frequency spectrum of added noise, $\mathrm{I}$ is input image.

Algorithm 1 recapitulates the employment of the projected SR explanation which is mentioned to as Fast Super Resolution (FSR) method in image domain and implementation of analytical solution.

Yest $=$ real (ifft(FR-FCBinv)* F2D * tau)

\section{B. Solution of the $L_{2}-L_{2}$ problem in the gradient domain}

Common image priors well-defined in the gradient province have remained effectively used for image refurbishment, eluding the communal ringing objects. In this portion, we emphasis on the gradient outline prior projected for the SISR problematic. This prior contains of in view of the normalizing term, therefore the problem chances to

$$
0.5 * \operatorname{resd}^{2}+\operatorname{tau} * \sum \operatorname{real}\left(\mathrm{x}-\mathrm{x}_{\mathrm{p}}\right)
$$

Where $\mathrm{x}$ is the low resolution image pixel, with $\mathrm{xp}$ as the input original image, where as resd is the residual pixel i.e is the difference between output high resolution image with tau as a constant. Here we check for the minimum of the above equation to get a good high resolution good image.

The gradient operatives can be rephrased as two matrices $D_{1}$ and $D_{2}$ equivalent to the horizontal and vertical discrete variances of an image, correspondingly. Consequently, two diagonal matrices are attained by disintegrating $\mathrm{D}_{1}$ and $\mathrm{D}_{2} \mathrm{in}$ the frequency domain, i.e.

$\mathrm{D} 1=\mu *$ ifft $(\mathrm{FB} * \mathrm{FX})$

$\mathrm{D} 2=\mu * \operatorname{ifft}(\mathrm{FB} * \mathrm{FX}+\mathrm{STy})$

With $\mathrm{A}=\left[\mathrm{D}^{\mathrm{T}}{ }_{1}, \mathrm{D}^{\mathrm{T}}{ }_{2}\right] \in \mathrm{R}$ and expending the notation $\nabla \mathbf{x}=\mathbf{v}=\left[\mathbf{v}_{\mathbf{h}}, \mathbf{v}_{\mathbf{v}}\right]^{\mathbf{T}} \in \mathrm{R}$. Note that the inevitability of $\mathrm{A}^{H} \mathrm{~A}$ is disrupted here since of the periodic border line supposition. Thus, totaling a small $\mathrm{L}_{2}$-norm regularization can sidestep this inevitability delinquent while possession the explanation closes to the innovative regularization.

Algorithm 2: FSR with gradient-domain $\mathrm{L}_{2}$ regularization implementation of the analytical solution of

$$
\text { Yest }=\text { real (ifft(FR-FCBinv) } * \text { F2D * tau })
$$

\section{Universal procedure of the proposed algorithm}

In instruction to custom the analytical explanation resulting for the $\mathrm{L}_{2}$-regularized $\mathrm{SR}$ problematic into an ADMM context, the delinquent is redrafted as the following inhibited optimization problem.

$\operatorname{Min}\left(0.5 * \operatorname{resd} 2+\operatorname{tau} * \sum \operatorname{real}\left(\mathrm{x}-\mathrm{X}_{\mathrm{p}}\right)\right)$

subject to $\mathrm{x}=\mathrm{u}$.

The AL purpose connected with this problematic is Objective $(\mathrm{t}, \mathrm{x}, \mathrm{d})=0.5 * \operatorname{resd} 2+\operatorname{tau} * \sum \operatorname{real}\left(\mathrm{x}-\mathrm{X}_{\mathrm{p}}\right)$ 
To solve difficult, we need to diminish objective $(\mathrm{t}, \mathrm{x}, \mathrm{d})$ w.r.t. $\mathrm{t}$ and $\mathrm{x}$ apprise the scaled dual in constant d iteratively as concise in Algorithm 3.

Memorandum that the 3rd step apprising the HR image $\mathrm{x}$ can be answered systematically using Theorem1. The in constant $u$ is restructured at the 4th step by means of the Moreau propinquity operative whose description is specified by

Criterion $(\mathrm{i})=$ real $($ objective $(\mathrm{t}, \mathrm{i}+1)-$ objective $(\mathrm{t}, \mathrm{i}) /$ objective $(\mathrm{t}, \mathrm{i}))$

\section{RESULTS}

In the simulation part the performance of the proposed super-resolution algorithms are evaluated both visually and quantitatively in terms of PSNR (Peak signal to noise ratio), ISNR (Improved signal to noise ratio), MSSIM (Mean structural similarity), Iterations, and time in seconds.

The below figure shows the simulation result of the super resolution result of zebra image in figure 1 . In this there are four push buttons the first button shows the input image. The input image is taken by the test image folder. Second push button shows the input low sample image which is converted into frequency domain from the time domain. The third push button shows the analytical solution which is the basic step to find super-resolution image. To implement this analytical solution 12-12 algorithm is used that is push button four shows the 12-12 algorithm resultant image of zebra. In this figure the PSNR value of analytical solution obtained as $30.078060 \mathrm{db}$ and the proposed algorithm shows the $30.078126 \mathrm{db}$. And the ISNR value of analytical solution shows the $1.4635 \mathrm{db}$ and proposed method shows as $6.1617 \mathrm{db}$.

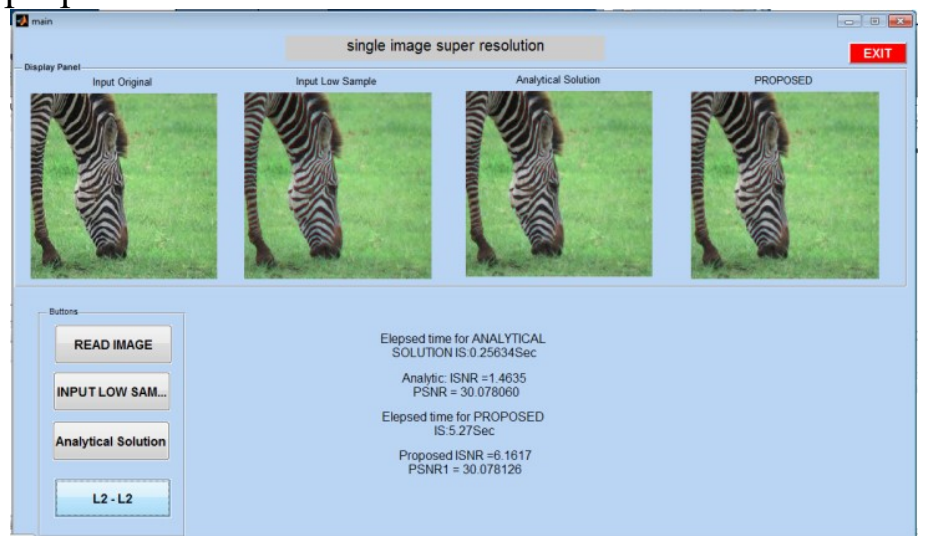

Figure 1 Super- resolution of zebra image simulation result.
Figure 2 shows the proposed method result, in this the iterations is the 142 and the time is $5.27 \mathrm{sec}$. The SSIM value is the $0.8804 \mathrm{db}$ as shown in the command window.

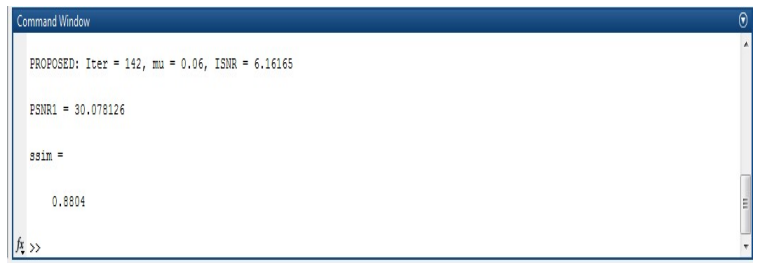

Figure 2 Proposed method result of zebra image in command window.

The below figure shows the simulation result of the super-resolution result of pepper image in figure 3. In this there are four push buttons the first button shows the input image. The input image is taken by the test image folder. Second push button shows the input low sample image which is converted into frequency domain from the time domain. The third push button shows the analytical solution which is the basic step to find super-resolution image. To implement this analytical solution 12-12 algorithm is used that is push button four shows the 12-12 algorithm resultant image of pepper.

In this figure the PSNR value of analytical solution obtained as $34.194068 \mathrm{db}$ and the proposed algorithm shows the $34.194139 \mathrm{db}$. And the ISNR value of analytical solution shows the $1.2221 \mathrm{db}$ and proposed method shows as $18.39 \mathrm{db}$.

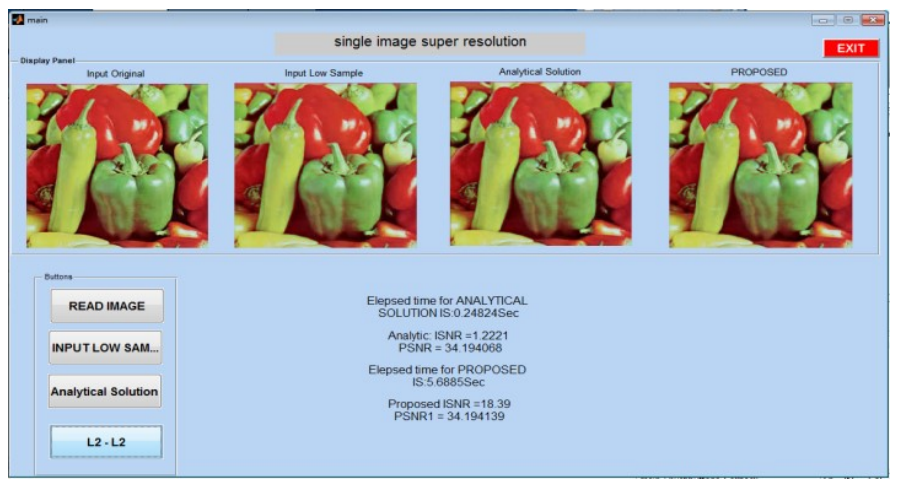

Figure 3 Super- resolution of pepper image simulation result.

Figure 4 shows the proposed method result, in this the iterations is the 147 and the time is $5.6885 \mathrm{sec}$. The SSIM value is the $0.9136 \mathrm{db}$ as shown in the command window.

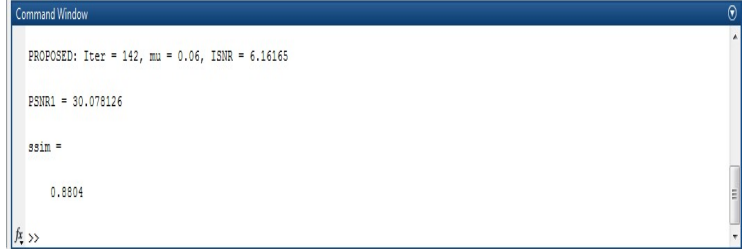

Figure 4 Proposed method result of zebra image in command window. 
These results are compared with the existing methods as shown in below table 1 .

Table 1. Super-resolution of the Lena and Barbara images compared with the existing method.

\begin{tabular}{|c|c|c|c|c|c|c|}
\hline Image & Method & $\begin{array}{c}\text { PSNR } \\
(\mathrm{db})\end{array}$ & $\begin{array}{c}\text { ISNR } \\
(\mathrm{db})\end{array}$ & $\begin{array}{c}\text { MSS } \\
\text { MM }\end{array}$ & $\begin{array}{l}\text { Time } \\
(\mathrm{sec})\end{array}$ & Iter \\
\hline \multirow[t]{3}{*}{ Zebra } & Bicubic & 18.98 & - & 0.37 & 0.001 & - \\
\hline & \begin{tabular}{|l}
$\mathrm{SC}$ [13] \\
\end{tabular} & 19.15 & 0.16 & 0.38 & 170.9 & \\
\hline & Proposed & 30.07 & 6.16 & 0.88 & 5.27 & 142 \\
\hline \multirow[t]{3}{*}{ Pepper } & Bicubic & 25.37 & $T$ & 0.48 & 0.002 & - \\
\hline & $\begin{array}{l}\text { ADMM } \\
{[13]}\end{array}$ & 29.26 & 4.01 & 0.67 & 1.92 & 隹 \\
\hline & Proposed & 34.19 & 18.39 & 0.91 & 5.688 & 147 \\
\hline
\end{tabular}

\section{CONCLUSION}

This paper studied a new fast single image superresolution framework based on the widely used image formation model. The proposed super-resolution approach computed the super resolved image efficiently by exploiting intrinsic properties of the decimation and the blurring operators in the frequency domain. A large variety of priors was shown to be able to be handled in the proposed super-resolution scheme. Specifically, when considering an 12regularization, the target image was computed analytically, getting rid of any iterative steps. For more complex priors (i.e., non 12-regularization), variable splitting allowed this analytical solution to be embedded into the augmented Lagrangian framework, thus accelerating various existing schemes for single image super-resolution.

\section{REFERENCES}

1. G. Martin and J. M. Bioucas-Dias, "Hyperspectral compressive acquisition in the spatial domain via blind factorization," in Proc. IEEE Workshop on Hyperspectral Image and Signal Processing: Evolution in Remote Sensing (WHISPERS), Tokyo, Japan, June 2015.

2. J. Yang and T. Huang, Super-resolution imaging. Boca Raton, FL, USA: CRC Press, 2010, ch. Image super-resolution: Historical overview and future challenges, pp. 20-34.

3. T. Akgun, Y. Altunbasak, and R. M. Mersereau, "Super-resolution reconstruction of hyperspectral images," IEEE Trans. Image Process., vol. 14, no. 11, pp. 1860-1875, 2005.

4. I. Yanovsky, B. H. Lambrigtsen, A. B. Tanner, and L. A. Vese, "Efficient deconvolution and super-resolution methods in microwave imagery," IEEE J. Sel. Topics Appl. Earth Observations and Remote Sens., vol. 8, no. 9, pp. 4273-4283, 2015.

5. R. Morin, A. Basarab, and D. Kouame, "Alternating direction method of multipliers framework for super-resolution in ultrasound imaging," in Proc. IEEE International Symposium on Biomedical Imaging (ISBI), Barcelona, Spain, May 2012, pp. 1595-1598.

6. R. L. Lagendijk and J. Biemond Iterative Identification and Restoration of Images. Boston, MA: Kluwer, 1991.

7. D. C. Youla, "Generalized image restoration by the method of alternating orthogonal projections," IEEE Trans. Circuits Syst., vol. CAS-25, pp. 694$702,1978$.

8. W. Yin, S. Osher, D. Goldfarb, and J. Darbon, "Bregman iterative algorithms for L1minimization with applications to compressed sensing," SIAM J. Imag. Sci., vol. 1, no. 1, pp. 143-168, 2008.

9. M. K. Ng, P. Weiss, and X. Yuan, "Solving constrained total-variation image restoration and reconstruction problems via alternating direction methods," SIAM J. Sci. Comput., vol. 32, pp. 2710-2736, 2010.

10. J. Sun, J. Sun, Z. Xu, and H.-Y. Shum, "Image super-resolution using gradient profile prior," in Proc. IEEE Conference on Computer Vision and Pattern Recognition (CVPR), Anchorage, AK, 2008, pp. 1-8.

11. J. Yang, J. Wright, T. S. Huang, and Y. Ma, "Image super-resolution via sparse representation," IEEE Trans. Image Process., vol. 19, no. 11, pp. 2861-2873, 2010.

12. D. Glasner, S. Bagon, and M. Irani, "Superresolution from a single image," in Proc. IEEE Int. Conf. Comp. Vision (ICCV), Kyoto, Japan, 2009, pp. $349-356$

13. M. K. Ng, P. Weiss, and X. Yuan, "Solving constrained total-variation image restoration and reconstruction problems via alternating direction methods," SIAM J. Sci. Comput., vol. 32, pp. 2710-2736, 2010. 